\title{
Vegetative Parameters and Fruit Yield per Plot as Influenced by Planting Density and Fertigation in Processing Tomato Varieties
}

\author{
Ch. Shanmukhi*, M.L.N. Reddy and A.V.D. Dorajee Rao \\ College of Horticulture, Dr. Y.S.R.H.U, Venkataramannagudem, \\ West Godavari (Dist.) - 534101 (Andhra Pradesh), India \\ *Corresponding author
}

\begin{abstract}
A B S T R A C T
\section{Keywords}

Tomato, Canopy spread, Primary branches, Yield, Planting density, Fertigation

Article Info

Accepted: 02 May 2018 Available Online: 10 June 2018

The highest plant height $(133.84 \mathrm{~cm})$, primary branches (13.29), canopy spread (38.37 $\mathrm{cm})$, leaves (41.90) and fruit yield per plot $(970.24 \mathrm{~kg})$ till 120 DAT was recorded by the var. Abhinav. Planting density at $60 \mathrm{~cm}$ x $60 \mathrm{~cm}\left(\mathrm{~S}_{2}\right)$ recorded significantly the highest number of primary branches $(13.48)$ and canopy spread $(38.43 \mathrm{~cm})$ followed by $75 \mathrm{~cm} \mathrm{x}$ $40 \mathrm{~cm}\left(\mathrm{~S}_{3}\right)$. The lowest number of primary branches $(11.99)$ and canopy spread $(37.55 \mathrm{~cm})$ was recorded by $120 \mathrm{~cm}$ x $40 \mathrm{~cm}$. Application of $180 \mathrm{~N}: 90 \mathrm{P}: 90 \mathrm{~K} \mathrm{~kg}$ per ha $\left(\mathrm{F}_{3}\right) \mathrm{The}$ highest plant height $(133.30 \mathrm{~cm})$, primary branches $(13.60)$, canopy spread $(40.74 \mathrm{~cm})$, leaves $(40.21)$ and fruit yield per plot $(1066.04 \mathrm{~kg})$ followed by $150 \mathrm{~N}: 75 \mathrm{P}: 75 \mathrm{~K} \mathrm{~kg}$ per ha $\left(\mathrm{F}_{2}\right)$. The lowest plant height $(114.47 \mathrm{~cm})$, primary branches $(12.25)$, canopy spread $(34.62$ $\mathrm{cm})$, leaves $(35.76)$ and fruit yield per plot $(710.84 \mathrm{~kg})$ was recorded by the application of 120N: 60P: $60 \mathrm{~K} \mathrm{~kg}$ per ha $\left(\mathrm{F}_{1}\right)$. Among three way interactions, the highest number of fruit yield per plot was recorded by the combination of variety Abhinav $+75 \mathrm{~cm} \mathrm{x} 40 \mathrm{~cm}\left(\mathrm{~S}_{3}\right)+$ 180N: 90P: 90K kg per ha $(1396.68 \mathrm{~kg})$.
\end{abstract}

\section{Introduction}

Tomato (Solanum lycopersicum), flowering plant of the nightshade family (Solanaceae), cultivated extensively for its edible fruits. Labelled as a vegetable for nutritional purposes, tomatoes are a good source of vitamin $\mathrm{C}$ and the phytochemical lycopene.

The fruits are commonly eaten raw in salads, served as a cooked vegetable, used as an ingredient of various prepared dishes, and pickled. Additionally, a large percentage of the world's tomato crop is used for processing; products include canned tomatoes, tomato juice, ketchup, puree, paste, and "sundried" tomatoes or dehydrated pulp.

\section{Materials and Methods}

The present investigation on the effect of variety, planting density, fertigation level on growth, flowering and yield in processing variety of tomato (Solanum lycopersicum L.) was conducted during the year 2016-17 at Jain irrigation systems ltd, Chittoor. The major objectives of the study were to find out the better variety, planting density, fertigation level for optimum growth, flowering and yield in tomato under agro climatic conditions of 
chittoor. The results obtained along with relevant discussion are presented in this paper.

\section{Results and Discussion}

\section{Plant height (cm)}

The data on plant height (Table 1) revealed that there were significant differences due to variety, planting density, fertigation level and their interactions. The main effects due to the above factors were found significant at 90 and 120 days after transplanting (DAT). Similarly, the interaction effects between varieties, planting density and fertigation level was also found significant at 90 and 120 days after transplanting (DAT). The fertigation level was significant at all growth stages of the crop.

Among the varieties Abhinav recorded the highest plant height $(133.84 \mathrm{~cm})$ at 120 DAT. Planting density at $120 \mathrm{~cm}$ x $40 \mathrm{~cm}\left(\mathrm{~S}_{1}\right)$ recorded significantly the highest plant height $(130.10 \mathrm{~cm})$ followed by $60 \mathrm{~cm}$ x $60 \mathrm{~cm}\left(\mathrm{~S}_{2}\right)$ $(123.94 \mathrm{~cm})$. The lowest plant height was recorded by the planting density at $75 \mathrm{~cm} \mathrm{x} 40$ $\mathrm{cm}\left(\mathrm{S}_{3}\right)(121.17 \mathrm{~cm})$. Similar observations were also reported by Raghav (2000) and Kumar (2001).

Application of $180 \mathrm{~N}: 90 \mathrm{P}: 90 \mathrm{~K} \mathrm{~kg}$ ha $\left(\mathrm{F}_{3}\right)$ recorded the highest plant height $(133.30 \mathrm{~cm})$ and the lowest plant height $(114.47 \mathrm{~cm})$ was recorded by the application of $120 \mathrm{~N}$ : 60P: $60 \mathrm{~K} \mathrm{~kg}$ ha $\left(\mathrm{F}_{1}\right)$. The plant height increased from $86.93 \mathrm{~cm}$ at 30 DAT to $125.07 \mathrm{~cm}$ at 120 DAT. Among the varieties Abhinav was found to show superior values throughout the study. This may be perhaps due to its inherent genetic potential compared to Alankar. These results are in conformity with those of Singh et al., (2005).

The combination of variety Abhinav, planting density at $120 \mathrm{~cm} \times 40 \mathrm{~cm}$ coupled with $\mathrm{F}_{3}$ level (180N: 90P: $90 \mathrm{~K} \mathrm{~kg}$ ha) exhibited superior performance which might be due to very less competition and a high level of nutrient availability.

\section{Number of primary branches per plant}

Significant differences were observed in the number of primary branches per plant (Table 2) due to planting density, fertigation levels and their interactions at different days after transplanting.

The main effect fertigation, two way interaction planting density $\mathrm{x}$ fertigation and three way interaction were found significant at 30, 60, 90 and 120 DAT.

Planting density at $60 \mathrm{~cm}$ x $60 \mathrm{~cm}\left(\mathrm{~S}_{2}\right)$ recorded significantly the highest number of primary branches (13.48) on par with $75 \mathrm{~cm} \mathrm{x}$ $40 \mathrm{~cm}\left(\mathrm{~S}_{3}\right)$ (13.28). The lowest number of primary branches was recorded by the planting density at $120 \mathrm{~cm} \mathrm{x} 40 \mathrm{~cm}\left(\mathrm{~S}_{1}\right)$ (11.99).

Application of $180 \mathrm{~N}$ : $90 \mathrm{P}: 90 \mathrm{~K} \mathrm{~kg}$ per ha $\left(\mathrm{F}_{3}\right)$ recorded the highest number of primary branches per plant (13.60) which was followed by $150 \mathrm{~N}$ : $75 \mathrm{P}$ : $75 \mathrm{~K} \mathrm{~kg}$ per ha $\left(\mathrm{F}_{2}\right)$ (12.92) on par with the application of $120 \mathrm{~N}$ : 60P: $60 \mathrm{~K} \mathrm{~kg}$ per ha $\left(\mathrm{F}_{1}\right)$ (12.25). Similar increase in number branches due to higher nutritional levels was also reported by Gireesh and Malabasari (2014).

An over view of the results on plant height and number of primary branches indicated that those parameters showed an inverse relationship in all the three factors under study which was also evident by the earlier studies like Ayub et al., (2011) who reported that the plant height was maximum but the number of primary branches was minimum at the highest population density. The results were contradictory to those of Khan et al., (2000) who reported significant effect of seed rates on plant height of rice bean. 


\begin{tabular}{|c|c|c|c|c|c|c|c|c|c|c|c|c|c|}
\hline & \multirow{4}{*}{$\begin{array}{l}\text { Fertigation } \\
\text { (C) }\end{array}$} & \multirow{2}{*}{\multicolumn{12}{|c|}{ Variety (A) }} \\
\hline \multirow{3}{*}{ Planting density (B) } & & & & & & & & & & & & & \\
\hline & & \multicolumn{3}{|c|}{$30 \mathrm{DAT}$} & \multicolumn{3}{|c|}{60 DAT } & \multicolumn{3}{|c|}{90 DAT } & \multicolumn{3}{|c|}{$120 \mathrm{DAT}$} \\
\hline & & Alankar & Abhinav & Mean & Alankar & Abhinav & Mean & Alankar & Abhinav & Mean & Alankar & Abhinav & Mean \\
\hline \multirow{4}{*}{$\begin{array}{l}\mathrm{S}_{1}(120 \mathrm{~cm} \times 40 \mathrm{~cm}) \\
\left(2.08 \text { plants per } \mathrm{m}^{2}\right)\end{array}$} & $\mathrm{F}_{1}(120 \mathrm{~N}: 60 \mathrm{P}: 60 \mathrm{~K})$ & 81.13 & 90.93 & 86.03 & 98.99 & 110.59 & 104.79 & 106.41 & 119.45 & 112.93 & 115.05 & 120.66 & 117.85 \\
\hline & $\mathrm{F}_{2}(150 \mathrm{~N}: 75 \mathrm{P}: 75 \mathrm{~K})$ & 86.41 & 84.93 & 85.67 & 109.67 & 103.79 & 106.73 & 115.96 & 131.24 & 123.60 & 120.71 & 132.98 & 126.84 \\
\hline & $\mathrm{F}_{3}(180 \mathrm{~N}: 90 \mathrm{P}: 90 \mathrm{~K})$ & 96.15 & 93.40 & 94.77 & 111.51 & 108.53 & 110.02 & 114.80 & 171.44 & 143.12 & 116.96 & 174.29 & 145.62 \\
\hline & Mean & 87.89 & 89.75 & 88.82 & 106.70 & 107.63 & 107.16 & 112.37 & 140.71 & 126.54 & 117.57 & 142.64 & 130.10 \\
\hline \multirow{4}{*}{$\begin{array}{l}\mathrm{S}_{2}(60 \mathrm{~cm} \times 60 \mathrm{~cm}) \\
\left(2.78 \text { plants per } \mathrm{m}^{2}\right)\end{array}$} & $\mathrm{F}_{1}(120 \mathrm{~N}: 60 \mathrm{P}: 60 \mathrm{~K})$ & 82.57 & 75.41 & 78.99 & 102.40 & 97.33 & 99.86 & 109.15 & 126.87 & 118.01 & 112.31 & 128.53 & 120.42 \\
\hline & $\mathrm{F}_{2}(150 \mathrm{~N}: 75 \mathrm{P}: 75 \mathrm{~K})$ & 84.82 & 92.24 & 88.53 & 97.83 & 108.91 & 103.37 & 103.15 & 139.43 & 121.29 & 103.35 & 142.48 & 122.91 \\
\hline & $\mathrm{F}_{3}(180 \mathrm{~N}: 90 \mathrm{P}: 90 \mathrm{~K})$ & 92.98 & 93.84 & 93.41 & 124.03 & 113.80 & 118.91 & 122.45 & 131.18 & 126.81 & 124.25 & 132.75 & 128.50 \\
\hline & Mean & 86.79 & 87.16 & 86.97 & 108.08 & 106.68 & 107.38 & 111.58 & 132.49 & 122.03 & 113.30 & 134.58 & 123.94 \\
\hline $\mathrm{S}_{3}(75 \mathrm{~cm} \mathrm{x} 40 \mathrm{~cm})$ & $\mathrm{F}_{1}(120 \mathrm{~N}: 60 \mathrm{P}: 60 \mathrm{~K})$ & 82.37 & 73.23 & 77.80 & 99.20 & 93.13 & 96.16 & 97.12 & 107.95 & 102.53 & 100.46 & 109.85 & 105.15 \\
\hline & $\mathrm{F}_{2}(150 \mathrm{~N}: 75 \mathrm{P}: 75 \mathrm{~K})$ & 84.25 & 89.50 & 86.87 & 105.32 & 103.23 & 104.27 & 114.20 & 147.59 & 130.89 & 115.91 & 149.29 & 132.60 \\
\hline & $\mathrm{F}_{3}(180 \mathrm{~N}: 90 \mathrm{P}: 90 \mathrm{~K})$ & 88.70 & 91.99 & 90.34 & 101.95 & 103.50 & 102.72 & 136.36 & 112.09 & 124.22 & 137.77 & 113.79 & 125.78 \\
\hline & Mean & 85.10 & 84.90 & 85.00 & 102.15 & 99.95 & 101.05 & 115.89 & 122.52 & 119.20 & 118.04 & 124.31 & 121.17 \\
\hline For Comparing variet & s (A) and Fertigation & & & & & & & & & & & & \\
\hline $\mathrm{F}_{1}(120 \mathrm{~N}$ & $50 \mathrm{P}: 60 \mathrm{~K})$ & 82.02 & 79.85 & 80.94 & 100.19 & 100.34 & 100.27 & 104.22 & 118.08 & 111.15 & 109.27 & 119.67 & 114.47 \\
\hline $\mathrm{F}_{2}(150 \mathrm{~N}$ & 75P:75K) & 85.16 & 88.88 & 87.02 & 104.27 & 105.30 & 104.79 & 111.10 & 139.41 & 125.26 & 113.32 & 141.58 & 127.45 \\
\hline $\mathrm{F}_{3}(180 \mathrm{~N}$ & $90 \mathrm{P}: 90 \mathrm{~K})$ & 92.60 & 93.07 & 92.84 & 112.49 & 108.61 & 110.55 & 124.53 & 138.23 & 131.38 & 126.32 & 140.27 & 133.30 \\
\hline & & 86.59 & 87.27 & 86.93 & 105.65 & 104.75 & 105.20 & 113.28 & 131.91 & 122.59 & 116.30 & 133.84 & 125.07 \\
\hline & & $S E m \pm$ & CD & $5 \%$ & $S E m \pm$ & CD & at $5 \%$ & $S E m_{ \pm}$ & CD & at $5 \%$ & $S E m \pm$ & CD & at $5 \%$ \\
\hline Vari & $y(A)$ & - & & $S$ & - & & VS & 0.61 & & .76 & 0.26 & & .77 \\
\hline Planting & ensity (B) & - & & IS & 1.75 & & .04 & 0.75 & & .16 & 0.33 & & .94 \\
\hline Fertig & ion $(\mathrm{C})$ & 1.77 & & 10 & 1.75 & & .04 & 0.75 & & .16 & 0.33 & & .94 \\
\hline & & - & & IS & - & & vS & 1.06 & & .06 & 0.46 & & .34 \\
\hline & & - & & $S$ & - & & VS & 1.30 & & .74 & 0.57 & & 64 \\
\hline & & - & & $S$ & - & & VS & 1.06 & & .06 & 0.46 & & .34 \\
\hline $\mathrm{Ax}$ & $x \mathrm{C}$ & - & & $S$ & 4.30 & & 2.36 & 1.84 & & 30 & 0.80 & & .32 \\
\hline
\end{tabular}


Table.2 Number of primary branches as influenced by variety, planting density and fertigation in processing tomato

\begin{tabular}{|c|c|c|c|c|c|c|c|c|c|c|c|c|c|}
\hline \multirow[t]{3}{*}{ Planting density (B) } & \multirow{3}{*}{$\begin{array}{l}\text { Fertigation } \\
\text { (C) }\end{array}$} & \multicolumn{12}{|c|}{ Variety (A) } \\
\hline & & \multicolumn{3}{|c|}{$30 \mathrm{DAT}$} & \multicolumn{3}{|c|}{60 DAT } & \multicolumn{3}{|c|}{90 DAT } & \multicolumn{3}{|c|}{$120 \mathrm{DAT}$} \\
\hline & & Alankar & Abhinav & Mean & Alankar & Abhinav & Mean & Alankar & Abhinav & Mean & Alankar & Abhinav & Mean \\
\hline \multirow{4}{*}{$\begin{array}{l}\mathrm{S}_{1}(120 \mathrm{~cm} \times 40 \mathrm{~cm}) \\
\left(2.08 \text { plants per } \mathrm{m}^{2}\right)\end{array}$} & $\mathrm{F}_{1}(120 \mathrm{~N}: 60 \mathrm{P}: 60 \mathrm{~K})$ & 5.47 & 6.33 & 5.90 & 7.47 & 8.00 & 7.73 & 10.33 & 9.20 & 9.76 & 10.93 & 10.33 & 10.63 \\
\hline & $\mathrm{F}_{2}(150 \mathrm{~N}: 75 \mathrm{P}: 75 \mathrm{~K})$ & 8.87 & 7.20 & 8.03 & 9.73 & 11.40 & 10.56 & 10.60 & 12.27 & 11.43 & 11.80 & 13.40 & 12.60 \\
\hline & $\mathrm{F}_{3}(180 \mathrm{~N}: 90 \mathrm{P}: 90 \mathrm{~K})$ & 8.00 & 8.00 & 8.00 & 11.47 & 10.67 & 11.07 & 12.53 & 11.60 & 12.06 & 12.60 & 12.93 & 12.75 \\
\hline & Mean & 7.44 & 7.17 & 7.30 & 9.55 & 10.02 & 9.70 & 11.15 & 11.02 & 11.08 & 11.77 & 12.22 & 11.99 \\
\hline \multirow{4}{*}{$\begin{array}{l}\mathrm{S}_{2}(60 \mathrm{~cm} \times 60 \mathrm{~cm}) \\
\left(2.78 \text { plants per } \mathrm{m}^{2}\right)\end{array}$} & $\mathrm{F}_{1}(120 \mathrm{~N}: 60 \mathrm{P}: 60 \mathrm{~K})$ & 6.40 & 6.47 & 6.43 & 8.33 & 10.00 & 9.16 & 9.13 & 11.00 & 10.06 & 10.60 & 11.67 & 11.13 \\
\hline & $\mathrm{F}_{2}(150 \mathrm{~N}: 75 \mathrm{P}: 75 \mathrm{~K})$ & 7.73 & 4.93 & 6.33 & 14.93 & 9.13 & 12.03 & 15.87 & 12.47 & 14.17 & 16.00 & 13.20 & 14.60 \\
\hline & $\mathrm{F}_{3}(180 \mathrm{~N}: 90 \mathrm{P}: 90 \mathrm{~K})$ & 8.60 & 6.60 & 7.60 & 12.67 & 13.73 & 13.20 & 14.33 & 14.47 & 14.40 & 14.13 & 15.33 & 14.73 \\
\hline & Mean & 7.57 & 6.00 & 6.78 & 11.97 & 10.95 & 11.46 & 13.11 & 12.64 & 12.87 & 13.57 & 13.40 & 13.48 \\
\hline \multirow{4}{*}{$\begin{array}{l}\mathrm{S}_{3}(75 \mathrm{~cm} \mathrm{x} 40 \mathrm{~cm}) \\
\left(3.33 \text { plants per } \mathrm{m}^{2}\right)\end{array}$} & $\mathrm{F}_{1}(120 \mathrm{~N}: 60 \mathrm{P}: 60 \mathrm{~K})$ & 6.67 & 6.67 & 6.67 & 9.27 & 12.93 & 11.10 & 9.93 & 15.40 & 12.66 & 10.87 & 16.20 & 11.53 \\
\hline & $\mathrm{F}_{2}(150 \mathrm{~N}: 75 \mathrm{P}: 75 \mathrm{~K})$ & 7.73 & 5.40 & 6.56 & 13.00 & 9.80 & 11.4 & 13.33 & 10.93 & 12.13 & 13.87 & 12.20 & 13.03 \\
\hline & $\mathrm{F}_{3}(180 \mathrm{~N}: 90 \mathrm{P}: 90 \mathrm{~K})$ & 7.60 & 5.93 & 6.76 & 11.00 & 13.73 & 12.36 & 11.53 & 16.47 & 14.00 & 12.20 & 14.40 & 13.30 \\
\hline & Mean & 7.33 & 6.00 & 6.66 & 11.09 & 12.15 & 11.62 & 11.59 & 14.26 & 12.92 & 12.31 & 14.26 & 13.28 \\
\hline \multicolumn{14}{|c|}{ For Comparing varieties (A) and Fertigation (C) } \\
\hline \multicolumn{2}{|c|}{$\mathrm{F}_{1}(120 \mathrm{~N}: 60 \mathrm{P}: 60 \mathrm{~K})$} & 7.66 & 6.35 & 7.01 & 10.35 & 10.40 & 10.37 & 11.02 & 11.40 & 11.21 & 12.08 & 12.42 & 12.25 \\
\hline \multicolumn{2}{|c|}{$\mathrm{F}_{2}(150 \mathrm{~N}: 75 \mathrm{P}: 75 \mathrm{~K})$} & 6.62 & 5.97 & 6.30 & 10.55 & 10.02 & 10.28 & 12.04 & 12.35 & 12.20 & 12.60 & 13.24 & 12.92 \\
\hline \multicolumn{2}{|c|}{$\mathrm{F}_{3}(180 \mathrm{~N}: 90 \mathrm{P}: 90 \mathrm{~K})$} & 8.06 & 6.84 & 7.45 & 11.71 & 12.71 & 12.21 & 12.80 & 14.17 & 13.48 & 12.97 & 14.22 & 13.60 \\
\hline \multicolumn{2}{|c|}{ Mean } & 7.45 & 6.39 & 6.92 & 10.87 & 11.04 & 10.95 & 11.95 & 12.64 & 12.29 & 12.55 & 13.29 & 12.92 \\
\hline \multicolumn{2}{|c|}{ Factors } & \multicolumn{3}{|c|}{ CD at $5 \%$} & $S E m \pm$ & \multicolumn{2}{|c|}{ CD at $5 \%$} & \multicolumn{3}{|c|}{ CD at $5 \%$} & $S E m_{ \pm}$ & \multicolumn{2}{|c|}{ CD at $5 \%$} \\
\hline \multicolumn{2}{|c|}{ Variety (A) } & \multicolumn{3}{|c|}{0.45} & - & \multicolumn{2}{|c|}{$N S$} & \multicolumn{3}{|c|}{$N S$} & - & \multicolumn{2}{|c|}{$N S$} \\
\hline Planting $\mathrm{c}$ & ensity (B) & - & & $\checkmark S$ & 0.14 & & 42 & 0.37 & & .08 & 0.33 & & 97 \\
\hline Fertiga & ion $(\mathrm{C})$ & 0.19 & & 55 & 0.14 & & 42 & 0.37 & & .08 & 0.33 & & 97 \\
\hline A & & - & & $\checkmark S$ & 0.20 & & .59 & 0.53 & & .52 & - & & IS \\
\hline & & 0.33 & & 96 & 0.25 & & 73 & 0.65 & & .87 & 0.58 & & 68 \\
\hline A & & - & & $\checkmark S$ & 0.20 & & .59 & - & & $\mathrm{VS}$ & - & & IS \\
\hline $\mathrm{AX}$ & $\mathrm{XC}$ & 0.47 & & 36 & 0.36 & & 03 & 0.92 & & .64 & 0.83 & & 38 \\
\hline
\end{tabular}


Table.3 Canopy spread $(\mathrm{cm})$ as influenced by variety, planting density and fertigation in processing tomato

\begin{tabular}{|c|c|c|c|c|c|c|c|c|c|c|c|c|c|}
\hline \multicolumn{14}{|c|}{ Table.3 Canopy spread $(\mathrm{cm})$ as influenced by variety, planting density and fertigation in processing tomato } \\
\hline \multirow[t]{3}{*}{ Planting density (B) } & \multirow{3}{*}{$\begin{array}{l}\text { Fertigation } \\
\text { (C) }\end{array}$} & \multicolumn{12}{|c|}{ Variety (A) } \\
\hline & & \multicolumn{3}{|c|}{$30 \mathrm{DAT}$} & \multicolumn{3}{|c|}{$60 \mathrm{DAT}$} & \multicolumn{3}{|c|}{$90 \mathrm{DAT}$} & \multicolumn{3}{|c|}{120 DAT } \\
\hline & & Alankar & Abhinav & Mean & Alankar & Abhinav & Mean & Alankar & Abhinav & Mean & Alankar & Abhinav & Mean \\
\hline \multirow{4}{*}{$\begin{array}{l}\mathrm{S}_{1}(120 \mathrm{~cm} \times 40 \mathrm{~cm}) \\
\left(2.08 \text { plants per } \mathrm{m}^{2}\right)\end{array}$} & $\mathrm{F}_{1}(120 \mathrm{~N}: 60 \mathrm{P}: 60 \mathrm{~K})$ & 12.45 & 12.46 & 12.45 & 23.44 & 23.79 & 23.61 & 30.22 & 36.34 & 33.28 & 30.61 & 32.76 & 31.68 \\
\hline & $\mathrm{F}_{2}(150 \mathrm{~N}: 75 \mathrm{P}: 75 \mathrm{~K})$ & 15.09 & 15.75 & 15.42 & 29.96 & 29.50 & 29.73 & 37.50 & 41.44 & 39.47 & 38.34 & 38.92 & 38.63 \\
\hline & $\mathrm{F}_{3}(180 \mathrm{~N}: 90 \mathrm{P}: 90 \mathrm{~K})$ & 18.16 & 18.01 & 18.05 & 33.09 & 32.59 & 32.84 & 41.89 & 41.98 & 41.93 & 42.18 & 42.51 & 42.34 \\
\hline & Mean & 15.23 & 15.40 & 15.31 & 28.83 & 28.62 & 28.72 & 36.53 & 39.92 & 38.22 & 37.04 & 38.06 & 37.55 \\
\hline $\mathrm{S}_{2}(60 \mathrm{~cm} \times 60 \mathrm{~cm})$ & $\mathrm{F}_{1}(120 \mathrm{~N}: 60 \mathrm{P}: 60 \mathrm{~K})$ & 11.53 & 11.50 & 11.51 & 24.43 & 24.26 & 24.34 & 29.20 & 38.24 & 33.72 & 34.06 & 34.82 & 34.44 \\
\hline$\left(2.78\right.$ plants per $\left.\mathrm{m}^{2}\right)$ & $\mathrm{F}_{2}(150 \mathrm{~N}: 75 \mathrm{P}: 75 \mathrm{~K})$ & 12.96 & 12.89 & 12.92 & 25.47 & 24.88 & 25.17 & 30.67 & 32.75 & 31.71 & 39.60 & 40.26 & 39.93 \\
\hline & $\mathrm{F}_{3}(180 \mathrm{~N}: 90 \mathrm{P}: 90 \mathrm{~K})$ & 16.31 & 16.66 & 16.48 & 26.73 & 27.07 & 26.90 & 33.87 & 48.14 & 41.00 & 39.86 & 41.99 & 40.92 \\
\hline & Mean & 13.60 & 13.68 & 13.64 & 25.54 & 25.40 & 25.47 & 31.24 & 39.71 & 35.51 & 37.84 & 39.02 & 38.43 \\
\hline $\mathrm{S}_{3}(75 \mathrm{~cm} \mathrm{x} 40 \mathrm{~cm})$ & $\mathrm{F}_{1}(120 \mathrm{~N}: 60 \mathrm{P}: 60 \mathrm{~K})$ & 14.88 & 14.71 & 14.79 & 24.60 & 25.29 & 24.94 & 34.50 & 34.21 & 34.35 & 36.63 & 38.86 & 37.74 \\
\hline$\left(3.33\right.$ plants per $\left.\mathrm{m}^{2}\right)$ & $\mathrm{F}_{2}(150 \mathrm{~N}: 75 \mathrm{P}: 75 \mathrm{~K})$ & 16.84 & 17.54 & 17.19 & 27.25 & 24.85 & 26.05 & 32.33 & 33.02 & 32.67 & 37.54 & 36.57 & 37.05 \\
\hline & $\mathrm{F}_{3}(180 \mathrm{~N}: 90 \mathrm{P}: 90 \mathrm{~K})$ & 20.85 & 20.12 & 20.48 & 30.05 & 31.07 & 30.56 & 38.04 & 38.69 & 38.36 & 39.25 & 38.71 & 38.98 \\
\hline & Mean & 17.52 & 17.45 & 17.48 & 27.30 & 27.07 & 27.18 & 34.95 & 35.30 & 35.12 & 37.80 & 38.04 & 37.92 \\
\hline For Comparing variet & s (A) and Fertigation & & & & & & & & & & & & \\
\hline $\mathrm{F}_{1}(120 \mathrm{~N}$ & $50 \mathrm{P}: 60 \mathrm{~K})$ & 12.95 & 12.89 & 12.92 & 24.15 & 24.44 & 24.29 & 31.30 & 36.26 & 33.78 & 33.76 & 35.48 & 34.62 \\
\hline $\mathrm{F}_{2}(150 \mathrm{~N}$ & $75 \mathrm{P}: 75 \mathrm{~K})$ & 14.96 & 15.39 & 15.17 & 27.55 & 26.41 & 26.98 & 33.50 & 35.73 & 34.61 & 38.49 & 38.58 & 38.53 \\
\hline $\mathrm{F}_{3}(180 \mathrm{~N}$ & $90 \mathrm{P}: 90 \mathrm{~K})$ & 18.43 & 18.26 & 18.35 & 29.95 & 30.24 & 30.09 & 37.93 & 42.93 & 40.43 & 40.42 & 41.06 & 40.74 \\
\hline & & 15.45 & 15.51 & 15.48 & 27.22 & 27.03 & 27.12 & 34.24 & 38.31 & 35.27 & 37.56 & 38.37 & 37.96 \\
\hline $\mathbf{F a}$ & ors & $S E m$ & & at $5 \%$ & $S E m=$ & & at $5 \%$ & $S E m \pm$ & CD & at $5 \%$ & $S E m_{-}$ & CI & at $5 \%$ \\
\hline Varic & $y(A)$ & - & & $N S$ & - & & VS & 1.01 & & 92 & - & & $N S$ \\
\hline Planting & ensity (B) & 0.07 & & 0.20 & 0.25 & & .74 & - & & VS & - & & $N S$ \\
\hline Fertige & ion $(\mathrm{C})$ & 0.07 & & 0.20 & 0.25 & & .74 & 1.24 & & .58 & 0.41 & & .19 \\
\hline & & - & & $N S$ & - & & VS & - & & VS & - & & NS \\
\hline & & 0.12 & & 0.35 & 0.44 & & 28 & - & & VS & 0.71 & & .06 \\
\hline & & 0.10 & & 0.28 & - & & VS & - & & VS & - & & NS \\
\hline A x & $x \mathrm{C}$ & 0.17 & & 0.50 & - & & VS & - & & VS & - & & NS \\
\hline
\end{tabular}


Table.4 Number of leaves per plant as influenced by variety, planting density and fertigation in processing tomato

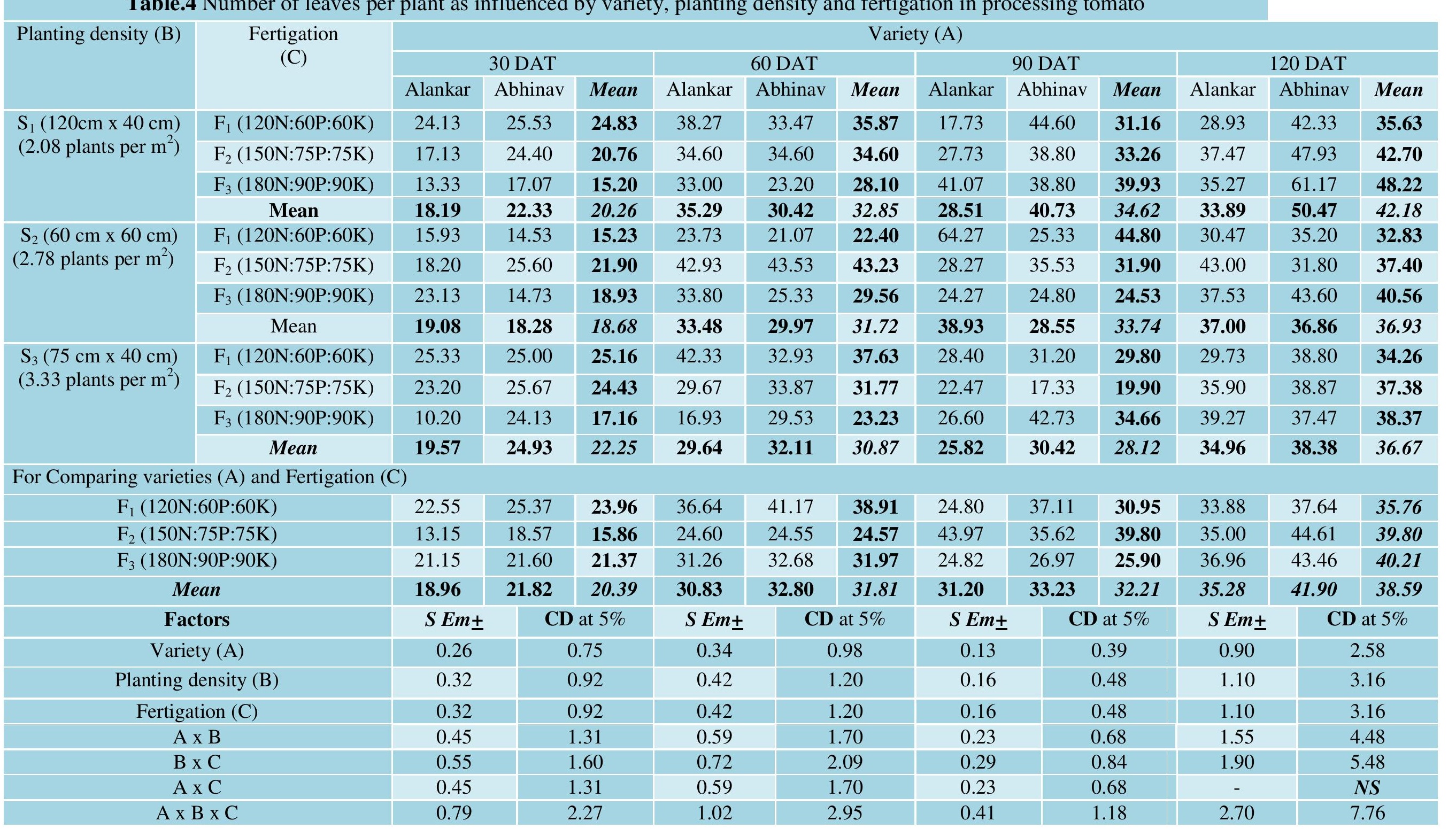




\begin{tabular}{|c|c|c|c|c|}
\hline \multirow{2}{*}{$\begin{array}{l}\text { Planting density } \\
\text { (B) }\end{array}$} & \multirow{2}{*}{$\begin{array}{l}\text { Fertigation } \\
\text { (C) }\end{array}$} & \multicolumn{3}{|c|}{ Variety (A) } \\
\hline & & Alankar & Abhinav & Mean \\
\hline \multirow{3}{*}{$\begin{array}{l}\mathrm{S}_{1}(120 \mathrm{~cm} \times 40 \mathrm{~cm}) \\
\left(2.08 \text { plants per } \mathrm{m}^{2}\right)\end{array}$} & $\mathrm{F}_{1}(120 \mathrm{~N}: 60 \mathrm{P}: 60 \mathrm{~K})$ & 535.46 & 646.60 & 591.03 \\
\hline & $\mathrm{F}_{3}(180 \mathrm{~N}: 90 \mathrm{P}: 90 \mathrm{~K})$ & 625.48 & $1,102.35$ & 863.91 \\
\hline & Mean & 591.16 & 922.73 & 756.94 \\
\hline \multirow{2}{*}{$\begin{array}{l}\mathrm{S}_{2}(60 \mathrm{~cm} \times 60 \mathrm{~cm}) \\
\left(2.78 \text { plants per } \mathrm{m}^{2}\right)\end{array}$} & $\mathrm{F}_{3}(180 \mathrm{~N}: 90 \mathrm{P}: 90 \mathrm{~K})$ & 860.85 & 957.32 & 909.09 \\
\hline & Mean & 826.82 & 859.96 & 843.39 \\
\hline \multirow{2}{*}{$\begin{array}{l}\mathrm{S}_{3}(75 \mathrm{~cm} \times 40 \mathrm{~cm}) \\
\left(3.33 \text { plants per } \mathrm{m}^{2}\right)\end{array}$} & $\mathrm{F}_{1}(120 \mathrm{~N}: 60 \mathrm{P}: 60 \mathrm{~K})$ & 680.90 & 915.71 & 798.31 \\
\hline & $\mathrm{F}_{2}(150 \mathrm{~N}: 75 \mathrm{P}: 75 \mathrm{~K})$ & 877.68 & $1,071.73$ & 974.71 \\
\hline \multicolumn{2}{|c|}{$\mathrm{F}_{2}(150 \mathrm{~N}: 75 \mathrm{P}: 75 \mathrm{~K})$} & 774.47 & $1,004.53$ & 889.50 \\
\hline \multicolumn{2}{|c|}{$\mathrm{F}_{3}(180 \mathrm{~N}: 90 \mathrm{P}: 90 \mathrm{~K})$} & 979.97 & $1,152.12$ & $1,066.04$ \\
\hline \multicolumn{2}{|c|}{ Mean } & 807.34 & 970.24 & \multirow{2}{*}{$\begin{array}{r}888.79 \\
\text { CD at } 5 \%\end{array}$} \\
\hline \multicolumn{2}{|c|}{ Factors } & \multicolumn{2}{|l|}{$S E m_{ \pm}$} & \\
\hline \multicolumn{2}{|c|}{ Variety (A) } & \multicolumn{2}{|l|}{2.77} & 7.97 \\
\hline \multicolumn{2}{|c|}{ Planting density (B) } & \multicolumn{2}{|l|}{3.39} & 9.76 \\
\hline \multicolumn{2}{|c|}{ Fertigation $(\mathrm{C})$} & \multicolumn{2}{|l|}{3.39} & 9.75 \\
\hline \multicolumn{2}{|c|}{$\mathrm{A} \times \mathrm{B}$} & \multicolumn{2}{|l|}{4.80} & 13.80 \\
\hline \multicolumn{2}{|c|}{$\mathrm{B} \times \mathrm{C}$} & \multicolumn{2}{|l|}{5.88} & 16.90 \\
\hline \multicolumn{2}{|c|}{$\mathrm{A} \times \mathrm{C}$} & \multicolumn{2}{|l|}{4.80} & 13.80 \\
\hline
\end{tabular}




\section{Canopy spread (cm)}

Data on canopy spread of a plant (Table 3) revealed significant differences due to fertigation levels at 30, 60, 90 and 120 DAT. However there was no significant difference in main the effects of variety and planting density. Application of $180 \mathrm{~N}$ : $90 \mathrm{P}: 90 \mathrm{~K} \mathrm{~kg}$ per ha $\left(\mathrm{F}_{3}\right)$ recorded the highest canopy spread (40.74) which was followed by $150 \mathrm{~N}$ : 75P: $75 \mathrm{~K} \mathrm{~kg}$ per ha $\left(\mathrm{F}_{2}\right)(38.53)$. The lowest canopy spread (34.62) was recorded by the application of $120 \mathrm{~N}: 60 \mathrm{P}: 60 \mathrm{~K} \mathrm{~kg}$ per ha $\left(\mathrm{F}_{1}\right)$.

\section{Number of leaves per plant}

Data on number of leaves per plant (Table 4) revealed significant differences due to variety, planting density, fertigation levels and their interactions. At 30, 60, 90 and 120 DAT, the three main effects and the three way interaction effects were significant, but the two way interaction effect of variety $x$ fertigation was non-significant. Among the main effects, the var. Abhinav recorded the highest number of leaves (41.90) at 120 DAT. Planting density at $120 \mathrm{~cm}$ x $40 \mathrm{~cm}\left(S_{1}\right)$ recorded significantly the highest number of leaves (42.18) followed by $60 \mathrm{~cm} \mathrm{x} 60 \mathrm{~cm}$ $\left(\mathrm{S}_{2}\right)$ (36.98) which was on par with the planting density at $75 \mathrm{~cm}$ x $40 \mathrm{~cm}\left(\mathrm{~S}_{3}\right)$ (36.67). Application of $180 \mathrm{~N}$ : $90 \mathrm{P}: 90 \mathrm{~K} \mathrm{~kg}$ per ha $\left(\mathrm{F}_{3}\right)$ recorded the highest number of leaves (40.21) which was on par with $150 \mathrm{~N}$ : 75P: $75 \mathrm{~K} \mathrm{~kg}$ per ha $\left(\mathrm{F}_{2}\right)(39.80)$. The lowest number of leaves (35.76) was recorded by the application of $120 \mathrm{~N}: 60 \mathrm{P}: 60 \mathrm{~K} \mathrm{~kg}$ per ha $\left(\mathrm{F}_{1}\right)$.

Plants which were widely spaced produced significantly more leaves and wider canopies. This might be because the wider spacing reduced the competition for soil nutrients, moisture, carbon dioxide and light among the plants. This probably enhanced photosynthesis which resulted in the production of more leaves and wider canopies
(Dawuda et al., 2011). Kamboj et al., (2015) also reported similar findings.

Likewise, the increase in the fertigation levels positively increased the plant height and canopy spread which was also supported by (Venkatesan et al., 2014). Similar findings were also reported by Odubanjo et al., (2011).

\section{Fruit yield per plot (kg)}

The fruit yield per plot (Table 5) exhibited significant differences due to variety, planting density, fertigation level and their interactions. Among the varieties Abhinav recorded the highest fruit yield per plot $(970.24 \mathrm{~kg})$. Planting density at $75 \mathrm{~cm} \times 40$ $\mathrm{cm}\left(\mathrm{S}_{3}\right)$ recorded significantly the highest fruit yield per plot $(1066.05 \mathrm{~kg})$ which was followed by $60 \mathrm{~cm}$ x $60 \mathrm{~cm}\left(\mathrm{~S}_{2}\right)(843.39 \mathrm{~kg})$ The lowest fruit yield per plot was recorded by the planting density at $120 \mathrm{~cm} \times 40 \mathrm{~cm}$ $\left(\mathrm{S}_{1}\right)(756.94 \mathrm{~kg})$. This might be due to higher plant population per unit area at narrow spacing. A positive correlation was reported between stand density and yield and negative one between stand density and individual plant productivity. These results are in agreement with Charlo et al., (2007). Application of 180N: 90P: $90 \mathrm{~K} \mathrm{~kg}$ per ha $\left(\mathrm{F}_{3}\right)$ recorded the highest fruit yield per plot (1066.04 kg) followed by $150 \mathrm{~N}: 75 \mathrm{P}: 75 \mathrm{~K} \mathrm{~kg}$ per ha $\left(\mathrm{F}_{2}\right)(889.50 \mathrm{~kg})$. The lowest fruit yield per plot $(710.84 \mathrm{~kg})$ was recorded by the application of $120 \mathrm{~N}$ : 60P: $60 \mathrm{~K} \mathrm{~kg}$ per ha $\left(\mathrm{F}_{1}\right)$.

The effect of variety, planting density and fertigation level was found significant on the fruit yield per plot. As it is observed in case of growth and fruit yield was found to be highest in case of Abhinav compared to Alankar establishing the superiority of the genotype.

However, there was significant difference between these three levels of planting density or population density with respect to fruit 
yield per plot. This may be due to the fact that even though an individual plant yielded more fruits at wider spacing, due to less number of plants per unit area, the net yield per unit area might had worked out to be lower compared to the case where there were more number of plants per unit area yielding lesser weight of fruits per plant. The marginal increase on per plant fruit number by reducing population did not compensate the marginal increase with elevated population levels per unit area.

Fertigation with the highest fertiliser dose made significant difference at all the population levels as compared to the lowest fertiliser doses. The additional dose of nutrients beyond the medium level resulted in a significant increase in the fruit yield per plot. The highest population density supplied with highest fertigation dose showed more fruit yield per plot. With every increase in population density, supply of additional dose of nutrients was found to be beneficial. It is leading to a point that a marginal increase in nutritional level was found beneficial with increased population but not so at the same level of population.

The experiment in the study was to find out the effect of variety, planting density and fertigation level on growth, flowering and yield in tomato. Plant height, number of leaves, number of primary and secondary branches and canopy spread were found to increase at a higher rate from 30 DAT to 90 DAT and at a lower rate from 90 DAT to 120 DAT. Among the varieties Abhinav exhibited superior values throughout the study which might be perhaps due to its inherent genetic potential compared to Alankar. There was highest average plant height with the planting density at $120 \mathrm{~cm} \times 40 \mathrm{~cm}\left(\mathrm{~S}_{1}\right)$ as compared to the higher densities of plants as in $60 \mathrm{~cm} \mathrm{x}$ $60 \mathrm{~cm}$ and $75 \mathrm{~cm} \mathrm{x} 40 \mathrm{~cm}$. As the fertilizer dose increased the vertical growth also increased and such an increase was statistically significant beyond recommended dose of $F_{2}$ level (150N:75P:75K) indicating a much balanced fertigation at $\mathrm{F}_{3}$ level (180N:90P:90K). So, maximum plant height was noticed at $\mathrm{F}_{3}$ level (180N:90P:90K). The combination of variety Abhinav, planting density at $120 \mathrm{~cm} \times 40 \mathrm{~cm}$ coupled with $\mathrm{F}_{3}$ level (180N:90P:90K) exhibited superior performance. The highest number of primary branches was noticed in the variety Abhinav compared to variety Alankar. Spacing at 60 $\mathrm{cm} \times 60 \mathrm{~cm}\left(\mathrm{~S}_{2}\right)$ that accommodated 2.78 plants per $\mathrm{m}^{2}$ exhibited the highest primary branching capacity which is on par with higher planting densities i.e., at $75 \mathrm{~cm} \times 40$ $\mathrm{cm}\left(\mathrm{S}_{2}\right)$ that accommodated 3.33 plants per $\mathrm{m}^{2}$. Application of $180 \mathrm{~N}: 90 \mathrm{P}: 90 \mathrm{~K}\left(\mathrm{~F}_{3}\right.$ level $)$ recorded the highest number of primary branches was significantly superior to the application of $150 \mathrm{~N}: 75 \mathrm{P}: 75 \mathrm{~K}\left(\mathrm{~F}_{2}\right.$ level). The highest canopy spread was noticed in the variety Abhinav compared to variety Alankar. Spacing at $60 \mathrm{~cm} \times 60 \mathrm{~cm}\left(S_{2}\right)$ that accommodated 2.78 plants per $\mathrm{m}^{2}$, exhibited the highest canopy spread than remaining planting densities. Application of $180 \mathrm{~N}: 90 \mathrm{P}: 90 \mathrm{~K}\left(\mathrm{~F}_{3}\right.$ level$)$ recorded the highest canopy spread was significantly superior to the application of $150 \mathrm{~N}: 75 \mathrm{P}: 75 \mathrm{~K}\left(\mathrm{~F}_{2}\right.$ level). The highest number of leaves was noticed in the variety Abhinav compared to variety Alankar.

A spacing of $120 \mathrm{~cm} \times 40 \mathrm{~cm}\left(\mathrm{~S}_{1}\right)$ that accommodated 2.08 plants per $\mathrm{m}^{2}$, exhibited the highest no of leaves than other planting densities. Application of 180N:90P:90K $\left(\mathrm{F}_{3}\right.$ level) recorded the highest number of leaves which is on par with the application of 150N:75P:75K $\left(\mathrm{F}_{2}\right.$ level). However, the interactions between planting density and fertigation level at per plot revealed that enhanced fertigation dose boosted the yield significantly from the lowest level 120N: 60P: $60 \mathrm{~K}$ to higher level $180 \mathrm{~N}$ : $90 \mathrm{P}: 90 \mathrm{~K} \mathrm{~kg}$ per ha. 


\section{References}

Ayub, M, Tahir, M, Ather Nadeem, M, Arif Zubair, M, Tariq, M. and Ibrahim, M. 2011. Effect of nitrogen applications on growth, forage yield and quality of three cluster bean varieties. Pakistan Journal of Life and Social Sciences. 8 (2): 11116.

Charlo, H.C.O, Castoldi, R, Ito, L.A, Fernandes, C. and Braz, L.T. 2007. Productivity of cherry tomatoes under protected cultivation carried out with different types of pruning and spacing. Acta Horticulturae. 761: 323-26.

Dawuda, M.M, Boateng, P.Y, Hemeng, O.B. and Nyarko G. 2011. Growth and yield response of carrot (Daucus carota L.) to different rates of soil amendments and spacing. Journal of Science and Technology. 31(2)11-20.

Gireesh, S. P. and Malabasari, T. A. 2014. Effect of major nutrient and picking stage on seed yield and quality of cluster bean (Cyamopsis tetragonoloba L. Taub). Research and Reviews: Journal of Agriculture and Allied Sciences. 3 (4): 8-12.

Kamboj, N.K. and Sharma, H.D. 2015. Effect of planting time and spacing on maturity, growth and fruit yield of bell pepper. International Journal of Farm Sciences. 5: 17-23.
Khan, H, Malik, M. A, Saleem, M. F, Cheema, M. A. and Ahmad, A. 2000. International Journal of Agricultural Biology. 1: 104-06.

Kumar, S. 2001. Response of hybrid tomato (Lycopersicon esculentum Mill.) to plant geometry and training on growth, yield and quality. M.Sc, Thesis, RAU, Bihar.

Odubanjo, O, Olufayo, A.A. and Oguntunde, P.G. 2011. Water use, growth and yield of drip irrigated cassava in a humid tropical environment. Soil Water Res. 6(1): 10-20.

Raghav, M. 2000. Effect of nitrogen and spacing on plant growth, dry matter accumulation and quality of hybrid tomato. Progressive Horticulture. 32: 90-94.

Singh, B.R. and Kumar, M. 2005. Effect of plant spacing and pruning on growth and yield of cherry tomato in greenhouse. Haryana Journal of Horticulture Science. 34: 179-80.

Venkatesan, K, Saraswathi, T, Pugalendhi, L. and Jansirani, P. 2014. Impact of Irrigation and Fertigation Levels on the Growth and Yield of Elephant Foot Yam (Amorphophallus paeoniifolius (Dennst.) Nicolson) Journal of Root Crops. 40(1). ISSN 0378-2409.

\section{How to cite this article:}

Shanmukhi, Ch., M.L.N. Reddy and Dorajee Rao, A.V.D. 2018. Vegetative Parameters and Fruit Yield per Plot as Influenced by Planting Density and Fertigation in Processing Tomato Varieties. Int.J.Curr.Microbiol.App.Sci. 7(06): 37-46. doi: https://doi.org/10.20546/ijcmas.2018.706.006 